\title{
La concepción hegeliana de la administración pública. Una sistematización de los aspectos principales del concepto de "estamento universal" (allgemeine Stand)
}

\author{
Eduardo Assalone
}

CONICET / Universidad Nacional de Mar del Plata, Argentina

\begin{abstract}
Resumen
El objetivo principal del presente artículo consiste en sistematizar la concepción hegeliana de la administración pública y evidenciar la originalidad de su propuesta. Para ello se presentan los distintos aspectos del "estamento universal" (allgemeine Stand) de acuerdo con el siguiente desarrollo. En primer lugar se expone la dimensión social del estamento universal, el hecho de que sea para Hegel uno de los tres estamentos (Stände) en los que se divide la sociedad civil (bürgerliche Gesellschaft). Luego se identifica la posición del estamento universal con respecto a la división social de los estamentos en base a un criterio lógico, el cual conduce a destacar la perspectiva universalista que deben poseer los administradores del Estado. Finalmente se explica la tarea de subsunción realizada por el estamento universal tomando como principio la relación igualmente lógica y "orgánica” entre los poderes del Estado.
\end{abstract}

\begin{abstract}
This papers attempts to systematize Hegel's understanding of public administration and to evince the originality of his approach. For that purpose we present different features of the "universal estate" (allgemeine Stand) as follows. First, we expound the social dimension of the universal estate, i.e., the fact that it is for Hegel one of the three estates (Stände) in which civil society (bürgerliche Gesellschaft) is divided. Then, we localize the universal estate in the social division of the estates on the basis of a logical criterion that leads us to highlight the universalistic point of view which civil servants must have. Finally, we explain the task of subsumption made by the universal estate, considering the logical and "organic" relation between the powers of the State as a principle.
\end{abstract}

\section{Introducción}

El "estamento universal” (allgemeine Stand) es quizás una de las figuras más complejas de la $P h R^{1}$ de Hegel. Primero por su dimensión social: es uno de los tres estamentos en los que se divide la sociedad civil pero, a diferencia de los otros dos, no se constituye en razón de la producción de bienes materiales (primarios o secundarios),
Palabras clave

\section{Hegel}

Filosofía del derecho administración pública estamento universal

\section{Keywords}

Hegel

Philosophy of right public administration universal estate 
2. Por "policía" no debemos entender el aparato represivo del Estado sino la administración pública, la mediación de la sociedad civil despolitizada y del Estado político. Cf. Riedel (1975: 266). El término

"Polizei" proviene de la Polizeiwissenschaft del antiguo cameralismo alemán. La "ciencia de la policía" es la "ciencia del gobierno". "Policía", en este contexto, designa el conjunto de disposiciones administrativas, políticas públicas y sociales, y los mecanismos de control sobre la actividad económica que le permiten al gobierno mantener un cierto orden y bienestar social. Sus amplias tareas son la protección del orden público, la inspección y planificación de la actividad industrial, la toma de medidas infraestructurales, garantizar la accesibilidad de los productos de primera necesidad a través de normativas impositivas y de diversificación, la provisión de instalaciones sanitarias, educativas y asistenciales. Cf. Horstmann (2005: 209). Sobre el origen y el concepto de la Polizei, véase: Maier (2009: 131 y ss), Knemeyer (1980), Aliscioni (2010, Cap. VII: 247-301). 3. CF. PhR, § 287, p. 457 [270]: “Esta tarea de subsunción [Subsumtion] en general concierne al poder gubernativo [Regierungsgewalt], en el cual están también comprendidos los poderes judicialy policial [die richterlichen und polizeilichen Gewalten], que se relacionan de modo inmediato con lo particular de

la sociedad civil y hacen valer en esos fines [particulares] el interés general." Traducción modificada. En todos los casos, los corchetes indican el número de página de la edición en español utilizada en las citas, según se consigna en la Bibliografía.

4. Véase: ibid., § 202 y ss.

5. Traducimos en todos los casos "allgemeine" por "universal" y no "general" a diferencia de la versión de Vermal. sino por su participación en el Estado. Segundo, por su dimensión política: el estamento universal conforma el poder gubernativo, es decir, no sólo es un estamento de la sociedad civil sino también un poder del Estado e incluso participa permanentemente de otro poder, el legislativo, integrando la asamblea de los estamentos. Tercero, por su relación con la policía y la justicia: el conjunto de funcionarios públicos que se ocupan de la administración pública (la policía, Polizei) ${ }^{2}$ y la administración de justicia (Rechtspflege) forman parte del estamento universal. A su vez, la policía y la justicia están organizadas bajo la dirección del poder gubernativo. ${ }^{3}$ Cuarto, por su composición heterogénea: como veremos, el estamento universal por supuesto está integrado por los miembros de la administración pública (y judicial), pero también lo integran los militares y hasta los "profesores" (Lehrer). Por último, aunque no de menor importancia, por el rol especial que cumple el estamento universal en la eticidad moderna: su "trabajo por lo universal" (Arbeit für das Allgemeine) (ibid., s 205, p. 357 [196]) se traduce, en el poder gubernativo, en una "subsunción de las esferas particulares y los casos individuales bajo lo universal" (Subsumtion der besonderen Sphären und einzelnen Fälle unter das Allgemeine) (ibid., S 273, p. 435 [254]).

En el presente artículo se desarrollan y sistematizan cada uno de estos aspectos, tomando en cuenta, como punto de partida, la dimensión social del estamento universal, el hecho de que sea para Hegel uno de los tres estamentos (Stände) en los que se divide la sociedad civil (bürgerliche Gesellschaft) (apartado 1). Luego se identifica la posición del estamento universal con respecto a la división social de los estamentos en base a un criterio lógico, el cual conduce a destacar la perspectiva universalista que deben poseer los administradores del Estado (apartado 2). Finalmente explicamos la tarea de subsunción realizada por el estamento universal tomando como principio la relación igualmente lógica y orgánica entre los poderes del Estado. En función de este desarrollo, el objetivo principal del presente artículo consiste en sistematizar la concepción hegeliana de la administración pública y evidenciar la originalidad de su propuesta.

\section{La dimensión social del estamento universal}

Quisiéramos analizar en primer lugar la dimensión social del estamento universal. Se trata de un estamento social y, por lo tanto, se encuentra afincado en la esfera de la sociedad civil. Por esta razón lo moviliza un interés particular como en el caso de los demás estamentos, el agrícola o "substancial" (substantielle) y el industrial o "formal" (formelle). ${ }^{4}$ Pero Hegel observa que ese interés particular coincide con el interés universal, de allí su denominación:

\begin{abstract}
El estamento universalse ocupa de los intereses universales de la situación social. Debe ser por lo tanto relevado del trabajo directo para satisfacer sus necesidades, por disponer de un patrimonio privado o por medio de una indemnización del Estado, que absorba, por otra parte, su actividad, de manera tal que el interés privado encuentre su satisfacción en su trabajo por lo universal (ibid., § 205, p. $357[196)^{5}$
\end{abstract}

El interés privado de los miembros del estamento universal encuentra su satisfacción en el trabajo por lo universal en nombre del Estado. De esta manera, lo particular sigue existiendo aunque identificado con lo universal. Asimismo, lo universal tampoco es "contaminado" por esta identificación, porque lo que prima es el interés universal. En términos pragmáticos, si el funcionario público no cumple con su trabajo por lo universal, tampoco obtendría ninguna retribución económica de parte del Estado. La identificación funciona, y el individuo recibe una retribución por ello, sólo en la medida en que el interés particular del funcionario quede efectivamente subsumido en lo universal. 
En la cita anterior Hegel indica que el estamento universal: "Debe ser relevado del trabajo directo para satisfacer sus necesidades". En esta exigencia se basa la necesidad del individuo de percibir un ingreso de parte del Estado. Si el Estado requiere de individuos específicos para alcanzar sus fines universales; si las particulares capacidades de ciertos individuos, acreditadas en exámenes públicos, son requeridas por el Estado para realizar la universalidad, entonces dichos individuos deben ocuparse exclusivamente de las tareas estatales encomendadas. De lo contrario se le estaría exigiendo un doble trabajo, uno para su propia subsistencia y otro para la del Estado. Por lo tanto, esos individuos deben recibir un ingreso por parte del Estado que garantice su subsistencia. Si esa garantía no estuviera dada, el Estado descuidaría uno de sus principios fundamentales: "lo universal no se cumple ni tiene validez sin el interés, el saber y el querer particular" (ibid., S 260, p. 407 [233]). Además, pondría en riesgo innecesariamente la eficiencia del individuo a cargo de una tarea pública.

Por otra parte, si el Estado no ofreciera una indemnización o un resarcimiento (Schadloshaltung) al individuo por su ocupación en las tareas administrativas, únicamente podrían ocuparse de ellas las personas con mayores ingresos privados. Pues, solamente aquellas personas que gozaran de un alto nivel de vida por su actividad privada (ya sea por renta agraria o por beneficios industriales) tendrían la suficiente holgura económica como para poder dedicarse a la función pública a tiempo completo. Esa circunstancia no sólo conduciría al Estado hacia una plutocracia, sino que éste no respetaría tampoco el principio de libre elección del estamento: sólo los ricos podrían ser parte del estamento universal. Recordemos lo que Hegel sostiene con toda claridad a este respecto: "cualquiera sea el estamento a que pertenece el indivi$d u o$, si bien influyen en ello condiciones naturales, el nacimiento y una serie de circunstancias, la determinación última y esencial radica sin embargo en la opinión subjetiva y en el arbitrio particular" (der subjektiven Meinung und der besonderen Willkür) (ibid., \$ 206, p. 358 [196]). De lo contrario, tendríamos una sociedad de castas y no un Estado racional. ${ }^{6}$ En este Estado no sólo se respeta el derecho del individuo a elegir su modo de vida sino que también se le otorga a éste una retribución en caso de optar por el servicio del Estado:

El servicio del Estado exige [...] el sacrificio de la arbitraria y personal satisfacción de fines subjetivos, y da justamente por ello el derecho de encontrarla en el cumplimiento del deber, pero sólo en él. En esto reside, desde esta perspectiva, la unión [Verknüpfung] del interés universal y particular que constituye el concepto y la solidez interna del Estado (ibid., §294, Obs., p. 462 [273]; traducción modificada.).

Quien decida servir al Estado deberá sacrificar la satisfacción de sus intereses particulares salvo a través de su servicio público. ${ }^{7}$ De esa forma se garantiza tanto el respeto a la particularidad como el cumplimiento de los fines universales del Estado. El individuo encuentra en el desempeño de su cargo la "riqueza y una segura satisfacción de su particularidad, y la liberación de su situación exterior y de su actividad oficial de toda otra dependencia o influencia subjetiva" (ibid., \& 294, p. 461 [273]). No necesita entonces preocuparse por su subsistencia o por su progreso y de esa manera puede ejercer su actividad oficial sin distraerse en asuntos ajenos a los intereses universales.

El funcionario tiene derecho a recibir entonces una indemnización del Estado debido a su actividad oficial. Pero ese derecho no siempre debe ser ejercido. Aquí existe cierta ambigüedad por parte de Hegel. Por un lado afirma: "Por la satisfacción asegurada de la necesidad particular se elimina la necesidad exterior que puede ocasionar que se busquen los medios para satisfacerla a expensas de la actividad pública y del deber" (ibid., S 294, Obs., pp. 462-463 [273). En otras palabras, Hegel cree que el
6. Véase: ibid., § 206, Obs., p. 358 [197]. En otro parágrafo Hegel señala explícitamente que "la única condición" para el acceso a cargos públicos es la prueba del conocimiento y la capacidad del individuo para desempeñarse en dicho cargo. Esta única condición "asegura a todo ciudadano la posibilidad de integrar el estamento universal". ibid., § 291, p. [272]. El subrayado es nuestro.

7. En este contexto, el de las obligaciones de los servidores públicos, el "sacrificio de la arbitraria y personal satisfacción de fines subjetivos" quiere decir fundamentalmente que quien elige sumarse al estamento universal debe postergar cualquier tipo de actividad privada motivada únicamente por fines egoístas (hacer fortuna, por ejemplo), en la medida en que dicha actividad sea incompatible con el correcto desempeño de las tareas públicas. 
8. Esta idea aparece en varias de las lecciones sobre Rechtsphilosophie. En la $R p h$ III aparece de forma casi idéntica a la versión de la $P h R$ recién citada: “Este estamento [i.e., el universal] debe ser relevado del trabajo directo por las necesidades, o bien a través del patrimonio privado o bien a través de una indemnización del Estado". Rph III, pp. 166-167. En la Rph IV Hegel analiza las ventajas y desventajas de que el miembro del estamento universal tenga un patrimonio privado. $R p h I V, \S 205$ pp. 195-196. En la Rph V plantea la misma disyuntiva que analizamos ya entre el patrimonio privado y la indemnización pública. Rph V, § 205, p. 632. Esto se repite en la $R p h V I$, sólo que aquí la indemnización aparece como "salario": "Quien trabaja para el Estado debe ser relevado fácilmente del trabajo particular para la satisfacción de sus necesidades, o bien a través de un patrimonio privado, como rentista o terrateniente, o bien a través de un salario [Besoldung]." Rph VI, § 205, p. 521.

9. Rph IV, § 202, p. 193. CF. $P h R$, §306, Agregado, p. 475 [283]: "Este estamento [i.e., el substancial] tiene un querer más independiente. El estamento de los propietarios de bienes raíces [der Stand der Güterbesitzer] se divide en su parte cultivada y en el estamento de los campesinos [Bauernstand]. A ambos se oponen el estamento industrial [Stand des Gewerbes], dependiente de la necesidad, y el estamento universal, dependiente esencialmente de Estado [vom Staat wesentlich abhängig]". riesgo de corrupción en la administración pública se elimina cuando se les asegura a los funcionarios la satisfacción de sus necesidades personales mediante una indemnización. Sin embargo, este filósofo sostiene -como vimos-que el estamento universal debe ser "relevado del trabajo directo para satisfacer sus necesidades, por disponer de un patrimonio privado o por medio de una indemnización del Estado" (ibid., $\int 205$, p. 357 [196]; el subrayado es nuestro). Es decir, que el Estado debe ofrecer una indemnización sólo a aquellos individuos que no posean un patrimonio privado lo suficientemente abundante o permanente como para poder desempeñar la función pública sin preocuparse por sus asuntos económicos personales. ${ }^{8}$

\section{El criterio lógico de la división estamental: el estamento universal y su perspectiva universalista}

Más arriba mencionábamos el hecho de que el estamento universal, a diferencia de los otros dos estamentos, no se constituía a partir de la producción de bienes materiales sino por su participación en el Estado. Ante esta peculiaridad podría decirse que el criterio de división de los estamentos en general no viene dado directamente por la participación en la producción económica sino por el origen de los ingresos percibidos. Si el origen de los ingresos de un individuo es el trabajo de la tierra, entonces el mismo pertenece al estamento substancial. Si el origen es, en cambio, la industria -la artesanía, la actividad fabril o el comercio-, entonces pertenece al estamento formal. Si, por último, su ingreso lo obtiene del trabajo en el Estado, entonces pertenece al estamento universal. En la Rph IV Hegel sostiene: "El primero [i.e., el primer estamento] vive de la naturaleza; el segundo, de los hombres; el tercero, de lo universal, de los intereses universales"?

Este nuevo criterio presenta una dificultad con respecto a los funcionarios públicos que no reciben dinero de parte del Estado por tener asegurado de forma privada su sustento. En otros términos, puede haber miembros del estamento universal que a la vez sean miembros del estamento substancial, por ejemplo. Son parte del estamento substancial en cuanto al origen de su patrimonio privado y en cuanto a la contribución al patrimonio general; pero son parte del estamento universal en lo relativo a su actividad pública. Este tipo de dificultades en la interpretación de la $P h R$ se deben en gran medida a lo poco detalladas que suelen ser por momentos las afirmaciones de Hegel sobre cuestiones muy puntuales. Se comprende esa falta de detalle porque la $P h R$ no pretende ser una suerte de manual de instrucciones para la edificación del Estado, sino que es un compendio sobre los principios filosóficos del derecho natural y la ciencia política y, como tal, debe mantener cierto grado de generalidad propio de una ciencia filosófica.

Nos interesa destacar a partir de esta dificultad hermenéutica el principio fundamentalmente ético (en el sentido de sittlich) e incluso lógico, pero de ninguna manera exclusivamente económico, que divide la sociedad civil hegeliana en tres estamentos. Hegel explícitamente sostiene: "De acuerdo con el concepto [nach dem Begriffe], los estamentos se determinan como estamento substancial o inmediato, estamento formal o reflexivo y finalmente estamento universal" (PhR, S 202, p. 355 [194]). Son las relaciones lógicas entre universalidad y particularidad, y entre inmediatez, mediación y reflexión, las que permiten comprender y justificar la división social en estamentos. Entre el estamento substancial y el estamento universal existe una relación idéntica a la que guardan entre sí la universalidad abstracta y la universalidad concreta. El tercer estamento tiene también lo substancial como fin, al igual que el primer estamento, "pero ya no de un modo inmediato" (aber nicht mehr auf unmittelbare Weise) (Rph III, p. 163). Lo que se produce entre uno y otro es la mediación (Vermittlung) -o la "reflexión"- del estamento formal, el de la actividad industrial y además, por supuesto, 
la propia mediación del estamento universal. Al igual que el segundo estamento, el tercero "tiene su subsistencia mediada por la habilidad propia y, como el primero, la tiene asegurada por el todo social" (Enz., III, S 528, p. 323 [545]). La seguridad y la estabilidad que poseen el estamento agrícola y el universal -el uno por la tierra, el otro por el Estado- son ajenas al estamento industrial. Pero como este último, el estamento de los funcionarios públicos también depende de su habilidad (Geschicklichkeit) para ganarse la vida. Sin conocimientos y capacidades particulares acreditados mediante

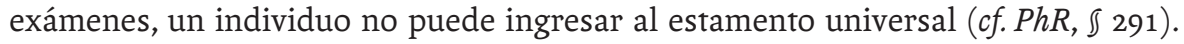
Del mismo modo, sin conocimientos y habilidades especiales, tampoco un individuo parece poder progresar en la actividad industrial.

En términos lógicos, el primer y tercer estamento comparten la universalidad, pero los diferencian la abstracción y la concreción o la inmediatez y la mediación, respectivamente. Esto último sucede también entre el primer y el segundo estamento: uno expresa la inmediatez que el otro mediatiza. Asimismo, el segundo y tercer estamento tienen en común la mediación, pero los separa la particularidad de los fines de uno frente a la universalidad de los fines del otro. Como podemos observar, Hegel expone los estamentos "según el concepto", y no según la estructura económica. ${ }^{10}$ En todo caso, la estructura económica manifiesta la misma trama dialéctica del concepto, pero no a la inversa.

Los estamentos sociales pueden incluso ser comprendidos como tres modos de conciencia: el conservadurismo, el individualismo y la universalidad (cf. Avineri, 2003: 156). Hegel dice expresamente que los estamentos son "sistemas particulares de necesidades, medios y trabajo, de modos de satisfacción y de cultura teórica y práctica [besonderen Systemen der Bedürfnisse, ihrer Mittel und Arbeiten, der Arten und Weisen der Befriedigung und der theoretischen und praktischen Bildung], en los que se reparten los individuos" (PhR, S 201, p. 354 [194]). Subrayamos lo siguiente: son sistemas particulares de cultura teórica y práctica. Esta Bildung no se reduce a la capacitación adquirida por el individuo en su actividad específica. También incluye los puntos de vista idiosincráticos de su estamento. Así, por ejemplo, Hegel ve mayor sentido de la individualidad y de la libertad en el estamento industrial que en el substancial, este último más ligado a la dependencia por su relación inmediata con la naturaleza. Por ello: "El primer estamento [i.e., el substancial] está, por lo tanto, más inclinado al sometimiento [Unterwürfigkeit] y el segundo [i.e., el industrial] más a la libertad" (ibid., S 204, p. 357 [196]). Pero ese sometimiento o conservadurismo también se vuelve una virtud para Hegel; lo cual se demuestra en la adjudicación de la cámara alta a los miembros del estamento substancial. ${ }^{11}$ Otro tanto sucede con el estamento universal: "Los miembros del gobierno y los funcionarios del Estado constituyen la parte principal del estamento medio [Mittelstandes], a la que pertenece la inteligencia culta [gebildete Intelligenz] y la conciencia jurídica de la masa de un pueblo" (ibid., s 297 , p. 464 [274]). El estamento universal tiene como especial punto de vista la cultura y la conciencia jurídica, lo cual lo hace especialmente apto para adoptar una perspectiva universalista, pues está cultivado en el concepto y en la ley.

Esta cultura del estamento universal incluso llega a ser bastante literal en la perspectiva hegeliana. En la Rph I, Hegel incluye a los "profesores" (Lehrer) en el estamento universal: "Al estamento universal pertenecen también los profesores, los cuales están al servicio del interés universal de las ciencias" (RphI, \ 105, Obs., p. 135). Los profesores comparten con los funcionarios de la administración pública y judicial un mismo punto de vista universal; en su caso, el de la ciencia. El papel de los funcionarios públicos parece ser entonces, para Hegel, una tarea no exclusivamente administrativa sino fundamentalmente intelectual (cf. Balibar, 2006: 59). Y así como todo funcionario es en algún sentido un intelectual, del mismo modo un profesor -o, en general, un intelectual- es también, de algún modo, un servidor del Estado. Tanto
10. Una prueba adicional de la estructura lógica de la división estamental surge del lugar que ocupa cada estamento en relación a los demás. En la Rph / Hegel afirma: "El estamento comercial constituye el estamento universal al interior del estamento industrial" (Der Handelsstand macht im Gewerbsstand den allgemeinen Stand aus) porque su ocupación consiste en la mediación (Vermittlung) que permite intercambiar los recursos (Mittel) superfluos de unos con los recursos superfluos de otros. Rph I, § 104, Obs., p. 134. Según esta afirmación, para definir el estamento depende de cuál sea la posición adoptada. Así, el estamento comercial, por su rol de intermediario, y por su visión universalista que le permite ver la equivalencia en lo disímil, puede ser considerado el estamento universal del estamento industrial.

11. Véase: ibid., §§ 305-307. 
12. Cf. Enz., III, § 545 , p. 346 [565]. Sobre el "estamento de la valentía" (Stande der Tapferkeit) o "estamento militar" (Militärstand) véase también: $P h R, \S \S$ $325 ; 326$, Obs:; 327, Agregado. el funcionario como el intelectual ejercen una misma función, la reconciliación de lo particular con lo universal; uno, en el ámbito de la administración pública; otro, en el de la ciencia. La $P h R$, como obra de un intelectual, es precisamente un ejemplo de esa tarea reconciliatoria en el campo de la ciencia política.

Parece entonces que el criterio para determinar la amplitud del estamento universal está dado por la adopción de una perspectiva universalista: la del gobierno, la de la ley, la de la autosuficiencia del Estado (en el caso de los militares) ${ }^{12}$ y la del concepto. Por eso este estamento es llamado por Hegel en la Enz. el "estamento pensante" (denkende Stand) (Enz., III, S 528, p. 323 [545]). Esa condición especial, "pensante", hace que sea especialmente apto para la función pública. Nos referimos ahora a su dimensión estrictamente política. Como decíamos antes, el estamento universal forma parte del Estado, no solamente de la sociedad civil. En el Estado compone el poder gubernativo (Regierungsgewalt), el ejecutivo o gobierno, la administración pública o, en un término que Hegel no utiliza, la burocracia (en un sentido no peyorativo). La cúspide de la estructura vertical del gobierno la ocupa el exponente mayor de otro poder del Estado, el monarca como poder del príncipe (fürstliche Gewalt). A su vez, tanto el poder gubernativo como el del príncipe participan de las asambleas del poder legislativo (gesetzgebende Gewalt). No hay en el Estado hegeliano un poder judicial independiente; la administración de justicia se encuentra en la sociedad civil pero en la órbita del poder gubernativo. El estamento universal también compone este último tipo de administración.

\section{El criterio lógico de la división de poderes: la tarea de subsun- ción del estamento universal}

Conviene contextualizar esta múltiple pertenencia institucional del estamento universal en el marco de la concepción hegeliana de la división de poderes. Hegel no acuerda con la concepción según la cual los poderes deben ser mutuamente independientes y funcionar como contrapeso uno del otro. Por cierto hay algo racional en la separación de los poderes del Estado que está directamente asociado al momento de diferencia al interior del concepto. Pero afirmar esa diferencia sin unidad, mantener la separación de un modo unilateral, es más bien, para Hegel, algo propio del "entendimiento abstracto" o "negativo", no de la razón: "El principio de la separación de los poderes contiene el momento esencial de la diferencia, de la racionalidad real [das wesentliche Moment des Unterschiedes, der realen Vernünftigkeit]; el entendimiento abstracto la aprehende en cambio falsamente como la absoluta independencia recíproca de los poderes, o bien unilateralmente, comprendiendo su relación como algo negativo, como mutua limitación [Beschränkung]" (PhR, S 272, Obs., p. 433 [253]). La constitución interna del Estado debe entonces presentar una división racional de los poderes donde éstos no se postulen como contrapesos necesarios, como momentos autónomos cuya relación es meramente negativa, sino como distintos aspectos de una misma totalidad "individual":

La constitución es racional en la medida en que el Estado determina y diferencia en sí su actividad de acuerdo con la naturaleza del concepto [nach der Natur des Begriffs]. Según ella, cada uno de los poderes es en sí mismo la totalidad, porque contiene en sí la actividad de los otros momentos y porque, al expresar éstos la diferencia del concepto, se mantienen en su idealidad y constituyen un único todo individual (ibid., § 272, p. 432 [252]).

La racionalidad de la constitución política es la racionalidad del concepto: en la medida en que aquélla exhiba en su diferenciación interna la naturaleza diferenciada del concepto, entonces será racional. En el concepto las diferencias no se excluyen 
mutuamente ni permanecen aisladas unas con respecto a otras (como en el caso del entendimiento). En el concepto cada momento particular es una totalidad que contiene a los otros momentos. El todo que reúne orgánicamente esos momentos o micrototalidades es a su vez una totalidad "individual", en el sentido de una unidad indivisible, como un organismo. ${ }^{13}$ El Estado monárquico-constitucional de Hegel es un organismo. ${ }^{14}$ La constitución política expresa la organicidad de ese Estado:

Este organismo [del Estado] es el desarrollo de la idea en orden en sus diferencias y en su realidad objetiva. Estos diferentes aspectos son así los distintos poderes y sus tareas y actividades, por medio de los cuales lo universal se produce continuamente y de un modo necesario-puesto que aquéllos están determinados por la naturaleza del concepto [durch die Natur des Begriffes]-, y al mismo tiempo se conserva, ya que está igualmente presupuesto a su producción. Este organismo es la constitución política (PhR, § 269, p. 414 [239]; traducción modificada).

El Estado es un organismo para Hegel porque despliega en sus poderes y en sus funciones lo que caracteriza a la idea. La idea se desarrolla a través de diferencias. Lo mismo sucede con el Estado en su división de poderes, y en la especialización de sus tareas y actividades. Ese desarrollo diferenciado produce continuamente la universalidad. Las diferencias sustentan la universalidad. La integración del Estado se produce porque su diferenciación está determinada por la naturaleza del concepto. No consiste en una diferenciación arbitraria y contingente sino necesaria. La constitución política es un organismo, pues en ella encontramos esa estructura a la vez diferenciada e integrada que caracteriza lo orgánico. La división de poderes debe derivar entonces de la articulación de una misma unidad (cf. Weisser-Lohmann, 2000: 162). Debe exhibir una estructura racional determinada por la naturaleza del concepto:

\section{Lo que encierra el origen absoluto de los diferentes poderes no es un fin o una utilidad cualquiera, sino únicamente la autodeterminación del concepto en sí mismo; sólo gracias a ella la organización del Estado es lo en sí mismo racional y la imagen de la razón eterna. En la lógica-y no por cierto en la lógica corriente- se sabe cómo el concepto y, más concretamente, la idea se determinan en sí mismos y ponen de modo abstracto sus momentos, la universalidad, la particularidad y la singularidad [Allgemeinheit, Besonderheit und Einzelheit] (PhR, $\S 272$, pp. 433-434 [253]). ${ }^{15}$}

Hegel busca justificar su división de poderes en la autodeterminación del concepto tal como la expuso en su Logik. Los momentos de la universalidad, la particularidad y la singularidad, así como también sus mediaciones recíprocas, deberán estar presentes en la diferenciación del Estado en poderes. De allí que en el parágrafo siguiente Hegel afirme que el poder legislativo es "el poder de determinar y establecer lo universal"; el poder gubernativo consiste en la "subsunción de las esferas particulares y los casos individuales bajo lo universal"; y el poder del príncipe representa "la subjetividad como decisión última de la voluntad" (ibid., S 273, p. 435 [254]). El momento de la universalidad corresponde al poder legislativo porque es éste el que produce la ley, cuya universalidad debe abstraerse necesariamente de cualquier determinación particular. Esta determinación corresponde al gubernativo en la medida en que subsume lo particular en lo universal (determinado y establecido justamente por el poder anterior), es decir, aplica la ley a situaciones particulares. Finalmente, el momento de la singularidad corresponde al príncipe, pues el mismo expresa en su persona la unidad individual (indivisible) del Estado a la que hacíamos referencia anteriormente. Sin ese momento de singularidad, es decir, de particularidad y universalidad al mismo tiempo, el Estado no sería para Hegel un organismo, pues funcionaría como una maquinaria inerte, carente de vida: “[...] la singularidad [del poder del príncipe] es al mismo tiempo lo que anima [die Seelenhaftigkeit], el principio vivificante [das
13. Para la concepción hegeliana del organismo, véase: Enz., II, §§ 337-376. Véase también: Beiser (2003), Itting (1987). 14. Hegel se refiere al Estado en numerosas oportunidades como si éste fuera un organismo. Véase: $P h R, \S 33 ;$; 256 , Obs.; §258, Agregado; § 259; § 263, Agregado; § 267 y § 267, Agregado; § 269 y $\S 269$, Agregado; § 271 y § 271, Agregado; § 276 Agregado; § 278, Obs.; § 286, Obs.; § 302 y $\S 302$, Obs.; $\S 308$, Obs. En virtud de ello puede decirse que la Rechtsphilosophie hegeliana representa una forma de organicismo político. Antecedentes de esta interpretación son, entre otros, los siguientes: Van Krieken (1873), McTaggart y McTaggart (1901: 177-196), Coker (1910). Desde una perspectiva crítica hacia la filosofía política de Hegel, la conocida obra de Karl Popper, La sociedad abiertay sus enemigos, también ha contribuido a la comprensión organicista de dicha filosofía. Véase: Popper (1947: 25-76). También han tenido gran influencia en este enfoque hermenéutico de la Rechtsphilosophie, las obras de Charles Taylor y Norberto Bobbio sobre este tema. Véase: Taylor (1977: 365388), Bobbio y Bovero (1979). En las últimas décadas, las contribuciones de Paolo Becchi, Frederick Beiser, Robert Pippin, Ludwig Siep y Michael Wolff, profundizan esta línea de interpretación. Véase: Becchi (1994), Beiser (2005: 224-258), Pippin (2008: 239-272), Siep (1986), Wolff (1984).
15. Como anteriormente, traducimos "Einzelheit" con "singularidad" en lugar de "individualidad" como hace Vermal. 
belebende Prinzip], la soberanía, que contiene en sí toda diferencia" (ibid., S 275, Agregado, p. 441 [258]). Lo distintivo del poder del príncipe radica entonces en la soberanía, "lo individual del Estado como tal" (ibid., \& 279, p. 444 [260]), la singularidad autodeterminada del Estado, tanto frente a los propios ciudadanos (soberanía interior) como a los otros Estados (soberanía exterior). ${ }^{16}$

Esa individualidad del Estado, correspondiente al momento de singularidad del concepto, sólo puede ser encarnada, según Hegel, por una persona, el monarca hereditario: "La personalidad del Estado sólo es efectivamente real como una persona, el monarca" (ibid., \279, Obs., p. [261]). Esta es, si se quiere, la mediación específica del príncipe: al ser toda disposición del Estado una decisión en última instancia suya; al ser toda voluntad universal -expresada en leyes, reglamentos, etc.- también una voluntad singular; el gobierno es un gobierno, el Estado es un Estado (cf. Bourgeois, 1989: 302). De alguna manera el estamento universal también necesita ser mediado: ${ }^{17}$ por ello el príncipe es la cabeza del poder gubernativo, porque sin él la administración pública -pero también las fuerzas armadas- sería, en el mejor de los casos, una maquinaria ciega y, en el peor, apenas un agregado de oficinas independientes que no merecerían el nombre de "Estado".

\section{Conclusión}

En su Rechtsphilosophie Hegel ubica en un lugar destacado a la administración pública. La necesidad de articular los intereses de los individuos y de los distintos sectores sociales con el interés universal custodiado por el Estado exige, en su concepción, la acción mediadora del estamento universal. El mismo se caracteriza por la complejidad de su posición particular y de su composición interna. En cuanto a lo primero, el estamento universal es uno de los tres estamentos en los que se divide la sociedad civil, pero también integra un poder del Estado (el gubernativo) y participa permanentemente de otro (el legislativo). En cuanto a lo segundo, el estamento universal no se compone únicamente de administradores públicos sino también de administradores de justicia, de militares e incluso de profesores. Esta composición compleja responde a un principio racional que revela el sentido conferido por Hegel al estamento universal: la adopción, por parte de los miembros de este estamento, de un punto de vista universalista -propio del Estado- del cual carece la sociedad civil.

De esta manera Hegel nos entrega una original teoría del Estado, de la relación de éste con la sociedad civil, y de la administración pública en particular. Entiende a esta última como una mediación necesaria de la eticidad moderna, como una articulación fundamental del "organismo" estatal. Si la mediación del poder legislativo es la mediación de lo universal, y la del poder del príncipe es la mediación de lo singular, la mediación del estamento universal es la correspondiente a lo particular. Esto significa que la "subsunción" administrativa incluye tanto la regulación de las diferentes actividades productivas como la aplicación de las resoluciones del príncipe o el cumplimiento de la ley. En el primer caso, lo particular de la actividad económica adquiere una dimensión universal, no sólo en el sentido de que comienza a ser regulada sino también en el sentido de que se articula armónicamente con las restantes actividades productivas por intermedio de la policía. En el segundo caso, la resolución del príncipe o la ley en su abstracción universalista adquieren mayor particularización, pues se aplican a esferas (por ejemplo en la administración de justicia). ${ }^{18} \mathrm{La}$ tarea del estamento universal incluye esa complejidad contenida en la subsunción: tanto la universalización de la voluntad particular como la particularización de la voluntad universal. 


\section{Abreviaturas de obras de G. W. F. Hegel}

Enz. Enzyklopädie der philosophischen Wissenschaften im Grundrisse.

Enz., II Enzyklopädie der philosophischen Wissenschaften im Grundrisse, Tomo 2, Die Naturphilosophie (ed. 1986).

Enz., III Enzyklopädie der philosophischen Wissenschaften im Grundrisse, Tomo 3, Die Philosophie des Geistes (ed. 1989).

Ilting Vorlesungen über Rechtsphilosophie 1818-1831, Edition und Kommentar in sechs Bänden von Karl-Heinz Ilting.

Logik Wissenschaft der Logik, en Werke in 20 Bänden.

PhR Grundlinien der Philosophie des Rechts, en Werke in 20 Bänden.

Rph I Vorlesungen über Rechtsphilosophie, Heidelberg, 1817-1818.

Rph III Vorlesungen über Rechtsphilosophie, Berlin, 1819-1820.

Rph IV Vorlesungen über Rechtsphilosophie, Berlin, 1821-1822.

Rph V Vorlesungen über Rechtsphilosophie, Berlin, 1822-1823.

Rph VI Vorlesungen über Rechtsphilosophie, Berlin, 1824-1825.

Werke Georg Wilhelm Friedrich Hegel Werke in 20 Bänden. 


\section{Bibliografía}

"Aliscioni, C. M. (2010). El capital en Hegel. Estudio sobre la lógica económica de la Filosofía del Derecho. Rosario: Homo Sapiens Ediciones.

" Avineri, S. (2003). Hegel's Theory of the Modern State. Cambridge: Cambridge University Press.

»Balibar, E. (2006). La filosofía de Marx (Trad. cast. de H. Pons). Buenos Aires: Nueva Visión.

" Becchi, P. (1994). La doctrina hegeliana del organicismo político (Trad. cast. de Esteban Federico Mizrahi). Escritos de Filosofía, 25-26, 75-99.

»Beiser, F. (2003). Hegel and Naturphilosophie, en Studies in History and Philosophy of Science, 34, 135-147.

》 Beiser, F. (2005). Hegel. New York: Routledge.

» Bobbio, N. y Bovero, M. (1979). Società e Stato nella filosofia politica moderna. Modello giusnaturalistico e modelo hegelo-marxiano. Milán: Il Saggiatore.

» Bourgeois, B. (1989). El príncipe hegeliano. En Amengual Coll, G. (Ed.). Estudios sobre la "Filosofía del Derecho" de Hegel (289-317). Madrid: Centro de Estudios Constitucionales.

» Brooks, T. (2007). No Rubber Stamp: Hegel's Constitutional Monarch. History of Political Thought XXVIII, 91-119.

"Coker, F. W. (1910), Organismic Theories of the State. Nineteenth Century Interpretations of the State as Organism or as Person. New York: Columbia University.

» Hegel, G.W.F. (1812-16).Wissenschaft der Logik, en dos tomos. En Werke, Bände 5-6.

» Hegel, G.W.F. (1817-18). Vorlesungen über Naturrecht und Staatswissenschaft Heidelberg 1817/18 mit Nachträgen aus der Vorlesung 1818/19 Nachgeschrieben von P. Wannenmann, Herausgegeben von C. Becker, W. Bonsiepen, A. Gethmann-Siefert, F. Hogemann, W. Jaeschke, Ch. Jamme, H.-Ch. Lucas, K. R. Meist, H. Schneider. En Hegel, G.W.F. (1983), Vorlesungen. Ausgewählte Nachschriften und Manuskripte, Band 1. Hamburg: Felix Meiner Verlag.

» Hegel, G.W.F. (1819-20). Philosophie des Rechts. Die Vorlesung von 1819/20 in einer Nachschrift, Herausgegeben von Dieter Henrich. Frankfurt a.M.: Suhrkamp, Erste Auflage.

" Hegel, G.W.F. (1821). Grundlinien der Philosophie des Rechts oder Naturrecht und Staatswissenschaft im Grundrisse, Mit Hegels eigenhändigen Notizen und den mündlichen Zusätzen, en: Werke, Band 7, 2. Aufl., 1989. Hegel, G.W.F. (2004), Principios de la Filosofía del Derecho (Trad. Juan Luis Vermal; 2da. ed). Buenos Aires: Sudamericana.

»Hegel, G.W.F. (1821-22). Die Philosophie des Rechts. Vorlesung von 1821/22, Herausgegeben von Hansgeorg Hoppe. Frankfurt a.M.: Suhrkamp, 2005.

"Hegel, G.W.F. (1822-23). Philosophie des Rechts. Nach der Vorlesungsnachschrift von H. G. Hotho 1822/23, en: Ilting, Band 3.

»Hegel, G.W.F. (1824-25). Philosophie des Rechts nach der Vorlesungsnachschrift K. G. v. Griesheims 1824-25. En Ilting, Band 4.

» Hegel, G.W.F. (1830). Enzyklopädie der philosophischen Wissenschaften im Grundris- 
se - 1830, en tres tomos. En: Werke, Bände 8-10. Trad. cast.: Hegel, G.W.F. (2000), Enciclopedia de las ciencias filosóficas en compendio (Trad. Ramón Valls Plana). Madrid: Alianza, 2000.

» Hegel, G.W.F. (1970). Georg Wilhelm Friedrich Hegel Werke [in 20 Bänden], auf der Grundlage der Werke von 1832-1845 neu edierte Ausgabe. Redaktion Eva Moldenhauer und Karl Markus Michel. Frankfurt a.M.: Suhrkamp Verlag.

» Hegel, G.W.F. (1973-74). Vorlesungen über Rechtsphilosophie 1818-1831, Edition und Kommentar in sechs Bänden von Karl-Heinz Ilting. Stuttgart-Bad Cannstatt: Frommann-Holzboog.

" Hegel, G.W.F. (1986). Enzyklopädie der philosophischen Wissenschaften im Grundrisse - 1830, en: Werke, Band 9, Zweiter Teil. Die Naturphilosophie. Mit den mündlichen Zusätzen, Erste Auflage. Hegel, G.W.F. (2000), Enciclopedia de las ciencias filosóficas en compendio (Trad. Ramón Valls Plana). Madrid: Alianza.

" Hegel, G.W.F. (1989). Enzyklopädie der philosophischen Wissenschaften im Grundrisse - 1830. En Werke, Band 10, Dritter Teil. Die Philosophie des Geistes. Mit den mündlichen Zusätzen, 2. Aufl. Hegel, G.W.F. (2000), Enciclopedia de las ciencias filosóficas en compendio (Trad. Ramón Valls Plana). Madrid: Alianza.

" Horstmann, R.-P. (2005). Hegels Theorie der bürgerlichen Gesellschaft. En Siep, L. (Eds.). G.W.F. Hegel. Grundlinien der Philosophie des Rechts (193-216). Berlin: Akademie Verlag, 2. Auf.

»Itting, K.-H. (1987). Hegels Philosophie des Organischen. En Petry, M.J. (Eds.). Hegel und die Naturwissenschaften (349-376). Stuttgart-Bad Cannstatt: FrommannHolzboog.

» Knemeyer, F.-L. (1980). Polizei. En Economy and Society 9 (2), 172-196.

》 Maier, H. (2009). Die ältere deutsche Staats- und Verwaltungs-Lehre. München: C.H. Beck.

" McTaggart, J. y McTaggart, E. (1901). Studies in Hegelian Cosmology. Cambridge: Cambridge University Press.

» Pippin, R.B. (2008). Hegel's Practical Philosophy. Rational Agency as Ethical Life. Cambridge: Cambridge University Press.

" Popper, K.R. (1947). The Open Society and Its Enemies, vol. II. The High Tide of Profecy: Hegel, Marx, and the Aftermath Londres: George Routledge \& Sons, Ltd.

» Riedel, M. (1975). Hegels Begriff der ,Bürgerlichen Gesellschaft' und das Problem seines geschichtlichen Ursprungs. En Ridel, M. (Ed.). Materialien zu Hegels Rechtsphilosophie Band 2 (247-275), Frankfurt a.M.: Suhrkamp.

» Siep, L. (1986). Hegels Theorie der Gewaltenteilung. En Lucas, H.-Ch. y Pöggeler, O. (Eds.). Hegels Rechtsphilosophie im Zusammenhang der europäischen Verfassungsgeschichte (387-420). Stuttgart-Bad Cannstatt: Frommann-Holzboog.

" Taylor, C. (1977). Hegel. Cambridge: Cambridge University Press.

»Van Krieken, A.T. (1873). Über die sogenannte organische Staatstheorie. Ein Beitrag zur Geschichte des Staatsbegriffs. Leipzig: Dunker und Humblot.

»Weisser-Lohmann, E. (2000). "Daß das Allgemeine zu einer Tat komme« - ıSittlichkeits und iVerfassung، bei Hegel. En Weisser-Lohmann, E. y Köhler, D. (Eds.). Verfassung und Revolution. Hegels Verfassungskonzeption und die Revolutionen der Neuzeit (137-166). Hamburg: Felix Meiner Verlag.

"Wolff, M. (1984). Hegels staatstheoretischer Organizismus: Zum Begriff und zur Methode der Hegelschen 'Staatswissenschaft. Hegel-Studien, 19, 147-177. 
